\title{
Targeting of MCT1 and PFKFB3 influences cell proliferation and apoptosis in bladder cancer by altering the tumor microenvironment
}

\author{
KE YAO HU ${ }^{1,2 *}$, DE GUI WANG ${ }^{3 *}$, PENG FEI LIU ${ }^{1 *}$, YAN WEI CAO ${ }^{1}$, YONG HUA WANG ${ }^{1}$, \\ XUE CHENG YANG ${ }^{1}$, CHENG XIA HU ${ }^{4}$, LI JIANG SUN ${ }^{1}$ and HAI TAO NIU ${ }^{1}$ \\ ${ }^{1}$ Department of Urology, The Affiliated Hospital of Qingdao University, Qingdao, Shandong 266101; ${ }^{2}$ Department of Urology, \\ The Hospital of Yantaishan, Yantai, Shandong 264000; ${ }^{3}$ Department of Anatomy, School of Basic Medical Sciences, \\ Lanzhou University, Lanzhou, Gansu 730000; ${ }^{4}$ Department of Pain Treatment, Haiyang Hospital of \\ The Affiliated Hospital of Qingdao University, Haiyang, Shandong 265100, P.R. China
}

Received January 13, 2016; Accepted February 26, 2016

DOI: $10.3892 / o r .2016 .4884$

\begin{abstract}
Phosphofructokinase-2/fructose-2,6-bisphosphatase 3 (PFKFB3) and monocarboxylate transporter 1 (MCT1) play important roles in tumor endothelial cells (ECs) and several biological processes. The present study was conducted to study the effects of PFKFB3 and MCT1 on cell proliferation and apoptosis in the tumor microenvironment by co-culture of HUVECs and T24, a bladder cancer (BC) cell line, using a microfluidic device. Immunofluorescence assay showed that HUVEC activity was significantly enhanced under co-culture with T24 cells, according to the stronger fluorescence intensity of CD31 and CD105 than that in the signal-cultured cells. Quercetin treatment inhibited MCT1 expression but did not affect PFKFB3 expression. Knockdown of MCT1 or/and PFKFB3 increased the apoptosis rate of the HUVECs under single-culture and co-culture situations by staining with calcein and propidium iodide. Meanwhile, cell proliferation and lactic concentration were significantly decreased after the blocking of MCT1 or/and PFKFB3, as compared with that in the control group. No obvious differences in the effects on apoptosis, proliferation and lactic concentration were found between cells treated with quercetin and siMCT1. Thus, we concluded that the targeting of MCT1 and PFKFB3 regulated cell proliferation and apoptosis in $\mathrm{BC}$ cells by altering the
\end{abstract}

Correspondence to: Dr Hai Tao Niu or Dr Li Jiang Sun, Department of Urology, The Affiliated Hospital of Qingdao University, Laoshan, 59 Haier Road, Qingdao, Shandong 266101, P.R. China

E-mail: niuht0532@126.com

E-mail: slijiang999@sohu.com

${ }^{*}$ Contributed equally

Key words: bladder cancer, tumor microenvironment, PFKFB3, MCT1, proliferation, apoptosis tumor microenvironment, and quercetin exhibited a potential antitumor effect by targeting MCT1.

\section{Introduction}

Bladder cancer (BC) is one of the most common cancers with high morbidity and mortality worldwide. BC seriously threatens human health and life (1). Endothelial cells (ECs) play critical roles in the tumor microenvironment and in angiogenesis, which are essential for the growth, proliferation and migration of cancer cells $(2,3)$. ECs are regarded as a significant target for antitumor angiogenesis and various anti-angiogenic drugs have been applied in the clinic (4). For this reason, identification of new molecules targeting ECs may become effective treatment strategies for $\mathrm{BC}$.

The Warburg effect states that cancer cells can secrete lactic acid (5). ECs are surrounded by a high lactic acid environment, which can activate numerous signaling pathways and promote EC proliferation (6,7). Recently, a study demonstrated that ECs rely on glycolysis for ATP production, and deletion of the phosphofructokinase-2/fructose-2,6-bisphosphatase 3 (PFKFB3) gene in ECs could suppress angiogenesis (8). PFKFB enzymes synthesize fructose-2,6-bisphosphate (F2,6P2), as an allosteric activator of PFK-1, an essential molecule in glycolysis and vascularization (9). As a single carboxylic acid transporter, monocarboxylate transporter 1 and 4 (MCT1 and MCT4) can transfer the lactic acid produced by tumor cells to the surrounding microenvironment, and then into ECs to cause a series of molecular events such as increased invasive activity (10). Targeting lactate metabolism has been reported to be studied in regards to cancer therapeutics, and PFKFB3 as well as MCTs have been studied as targets for cancer treatment $(9,11)$. However, the effects of PFKFB3 and MCT in the tumor microenvironment of $\mathrm{BC}$ remain unclear and warrant further research.

Three-dimensional (3D) co-culture models are constructed to simulate the cell in vivo environment when cells are cultured in vitro. The cells are grown within extracellular matrix (ECM) gels in a fluidic chip, and the chip is highly 
porous for seeding cells inside and providing large areas for cell-to-cell interactions (12). 3D co-culture allows cells to maintain normal shape, structure and function, in order to well reflect the microstructure, dynamic mechanical properties and biochemical functionalities to simulate a natural microenvironment (13). The microfluidic chip is an efficient experimental platform which unites numerous experiments into one chip and realizes 3D co-culture (14).

In the present study, in order to simulate the human tumor microenvironment, we conducted a co-culture with human umbilical vein endothelial cells (HUVECs) and human BC T24 cells, on a microfluidic chip. HUVEC activity was examined under a co-culture situation. The roles of PFKFB3 and MCT1 in cell proliferation, apoptosis and lactic acid synthesis were also investigated.

\section{Materials and methods}

Cell culture. HUVECs and T24, a BC cell line, were obtained from the Central Laboratory of the Affiliated Hospital of Qingdao University. The cells were cultured in Dulbecco's modified Eagle's medium (DMEM) (Gibco, Carlsbad, CA, USA) containing $10 \%$ fetal bovine serum (FBS), supplemented with $100 \mathrm{U} / \mathrm{ml}$ penicillin and $100 \mu \mathrm{g} / \mathrm{ml}$ streptomycin (Sigma, St. Louis, MO, USA) in a $37^{\circ} \mathrm{C}$ incubator containing $5 \% \mathrm{CO}_{2}$.

Preparation of matrix gel and microfluidic chip platform (co-culture and transfer chip). Firstly, the matrix gel was put into a refrigerator at $4^{\circ} \mathrm{C}$ overnight to enable it to thaw out completely. Then, the pre-cooled matrix gel was sufficiently mixed with DMEM (FBS-free) in a ratio of $1: 1$, and $10 \mu \mathrm{l}$ of mixed liquor was seeded into the central microchannel of the microfluidic chip lightly using a pipettor. The microfluidic chip was placed into a $37^{\circ} \mathrm{C}$ thermostatic incubator overnight to enable the matrix gel to solidify.

The microfluidic platform is exhibited in Fig. 1 and it has 3 major functions: co-culture of two types of cells, matrix gels between chambers to avoid mixed cells and high flux. The microfluidic chip has chambers and each chamber has two microchannels. The cells were seeded into chambers through one microchannel and the second channel ensures a stable pressure. Four chambers were separated by two major square crossing microchannels filled with Matrigel to simulate the membrane in the human body and made it easier to observe the behavior of a single type of cells. The chip was surrounded by a perfusion microchannel that could supply medium for cells in the chambers.

$3 D$ co-culture of HUVECs and T24. Three groups were designated in this experiment, namely control (HUVECs in complete medium), 2D (HUVECs co-cultured with T24 in complete solution) and 3D (HUVECs co-cultured with T24 in Matrigel) groups. In the 3D group, the suspension of HUVECs and T24 was prepared with a high density of $10^{7}$ cells $/ \mathrm{ml}$ and mixed with matrix gel in a proportion of $1: 1$. Then, the cell-matrix gel mixed suspension was separately seeded into two opposite chambers of the chip and the chip was cultured in a $37^{\circ} \mathrm{C}$ thermostatic incubator for $30 \mathrm{~min}$ to form a co-culture net. Meanwhile, complete culture solution was injected into the irrigation channel to support nutrition for cells in the
3D-matrix gel. The cells in the control and 2D groups were treated using the same method.

Immunofluorescence. After washing with phosphate-buffered saline (PBS), the cells were fixed with $4 \%$ paraformaldehyde in PBS and then permeabilized with $0.05 \%$ Triton X-100 in PBS. They were blocked with 3\% BSA for $2 \mathrm{~h}$, and stained with primary antibodies (anti-CD31, anti-CD105, anti-MCT1 or anti-PFKFB3; Abcam) at $4^{\circ} \mathrm{C}$ overnight. After washing with PBS, the chip was incubated with TRITC-conjugated secondary antibodies (BD, San Diego, CA, USA) for $40 \mathrm{~min}$, followed by staining with $1 \mu \mathrm{g} / \mathrm{ml}$ of DAPI (Sigma) for $5 \mathrm{~min}$ at room temperature. The chip was then rinsed with PBS 3 times and observed using fluorescence microscopy. Confocal imaging was performed using Zeiss 510 Meta system. The red and blue fluorescence was observed at 543 and $408 \mathrm{~nm}$, respectively.

Fluorescence cell viability assay. Cells were divided into 5 groups, namely the control group, quercetin (cells were treated with $5 \mu 10.02 \mathrm{nmol} / \mu 1$ quercetin), $\mathrm{MCT}^{\mathrm{KD}}$ group (MCT1 was knocked down with $5 \mu 10.02 \mathrm{nmol} / \mu 1 \mathrm{siMCT} 1$ ), PFKFB3 $^{\text {KD }}$ group (PFKFB3 was knocked down with $5 \mu 1$ $0.02 \mathrm{nmol} / \mu \mathrm{l}$ siPFKFB3) and $\mathrm{M}^{\mathrm{KD}}+\mathrm{P}^{\mathrm{KD}}$ group (MCT1 and PFKFB3 were both knocked down with $2.5 \mu 10.02 \mathrm{nmol} /$ $\mu 1 \mathrm{siMCT} 1$ and $2.5 \mu 10.02 \mathrm{nmol} / \mu 1 \mathrm{siPFKFB3).} \mathrm{The} \mathrm{control}$ group was treated with the same amount of DMEM. The siRNA treatment was performed according to the manufacturer's instructions when cells reached $60 \%$ confluency, and the second siRNAs were transfected $48 \mathrm{~h}$ later. Then, the cells were transferred into the microfluidic chip after $48 \mathrm{~h}$. After planting HUVECs into the microfluidic chip for 3 days, the cells were stained with $2 \mu \mathrm{mol} / 1$ calcein and $10 \mu \mathrm{mol} / 1$ propidium iodide to observe the cell apoptosis rate and death rate under fluorescence microscopy immediately. Conventional chemical synthesis of siRNAs as 21-25 nt of the double chain small molecule RNA was carried out by Guangzhou RiboBio Co., Ltd. (Guangzhou, China). Our previous study verified that they can effectively block MCT1 (15). In addition, the siRNAs for PFKFB3 were purchased from RiboBio; 3 pairs of different siRNAs were designed for different sites of the same target gene. The 3 pairs of siRNAs in accordance with a specific proportion were mixed into highly efficient silencing target gene products, that is, a cocktail.

Immunoblotting. The proteins were extracted from the lysates of the HUVECs with lysis buffer containing $20 \mathrm{mM}$ Tris- $\mathrm{HCl}$ (pH 7.5), $150 \mathrm{mM} \mathrm{NaCl}, 1 \mathrm{mM} \mathrm{Na} 2$ EDTA, $1 \mathrm{mM}$ EGTA, 1\% NP-40, 1\% sodium deoxycholate, $2.5 \mathrm{mM}$ sodium pyrophosphate and protease inhibitor. After centrifugation, the protein concentration was determined using the Bradford assay (Bio-Rad, Hercules, CA, USA). Approximately $20 \mu \mathrm{g}$ of the proteins was loaded and separated using $8 \%$ SDS-PAGE and transferred to a polyvinylidene difluoride (PVDF) membrane. Then, the members were blocked with 5\% BSA, followed by incubation with primary antibodies at $4{ }^{\circ} \mathrm{C}$ overnight. Immunodetection was accomplished using horseradish peroxidase-conjugated secondary antibody, followed by processing using an enhanced chemiluminescence (ECL) detection system. 



Figure 1. Image of the microfluidic chip for cell culture. The diameter is shorter than 1 yuan coin RMB $\sim 2 \mathrm{~cm}$. (A-D) Four chambers: 1, perfusion channel for nutrition support; 2 , channels for planting cells; 3 , central cross channel serving as membrane: a, entrance for inputting medium; b, exit for outputting medium; $\mathrm{c}$, entrance for inputting Matrigel; $\mathrm{d}$ and e, two holes for planting cells. In the present study, A and C were planted with HUVECs, while B and D were planted with T24 cells. Small molecules and factors can pass through the channels between the different chambers, and medium can supply energy to the cells from the surrounding perfusion channels.

Cell proliferation (CCK-8) assay. Cell proliferation of the HUVECs was evaluated using Cell Counting Kit-8 (CCK-8) assay (Dojindo) according to the manufacturer's instructions. Briefly, HUVECs were seeded into the microfluidic chip and co-cultured for 3 days. Then, the cells were digested and removed into a 96-well cell culture plate containing $100 \mu \mathrm{l}$ DMEM complete and grown for $8 \mathrm{~h}$ at $37^{\circ} \mathrm{C}$ with $5 \%$ $\mathrm{CO}_{2}$. After $8 \mathrm{~h}, 10 \mu \mathrm{l}$ CCK-8 reagents were added per well and incubation was carried out for $2 \mathrm{~h}$ in a $\mathrm{CO}_{2}$ incubator. Finally, $100 \mu 1$ supernatant from each well was transferred into fresh 96-well plates and the absorbance was measured at $450 \mathrm{~nm}$ using a spectrophotometer to calculate cell proliferation ability. Inhibition ratio $(\%)=(\mathrm{OD}$ ratio in the control group - OD ratio in the experimental group)/OD ratio in the control group x 100\%. The experiment was repeated 3 times.

Lactic concentration examination. Lactic concentrations in the different groups were examined using a lactate assay kit (BioVision) according to the manufacturer's instructions.

Statistical analysis. At least 3 separate experiments were performed for each measurement. All quantitative data are expressed as mean $\pm \mathrm{SD}$ and differences for comparisons were analyzed using t-test and one-way ANOVA followed by Tukey's post hoc test. All the analyses were performed using SPSS 19.0 software (SPSS, Inc., Chicago, IL, USA) and P $<0.05$ was considered to indicate a statistically significant result.

\section{Results}

Activation of HUVECs is detected in the co-culture condition. In order to detect the HUVEC activity in different conditions, immunofluorescence assay was conducted to detect CD31 and CD105 expression, which has been recognized as activity factors for ECs (16). As shown in Fig. 2A, CD31 and
CD105 showed the strongest fluorescence intensity in the 3D co-culture group and the weakest fluorescence intensity in the control group, and the expression levels of CD31 and CD105 in the $2 \mathrm{D}$ co-cultured group were higher than these levels in the control group and lower than those in the 3D co-culture group. Consistently, the results of western blotting showed similar expression levels, which reflected gradually increased expression of CD31 and CD105 from the control group, 2D co-culture to the 3D co-culture group (Fig. 2B).

Quercetin inhibits the activity of MCT1. Quercetin exists in flowers, leafs and fruits of many plants. As a natural inhibitor of MCT1, quercetin can inhibit MCT1 expression and then influence the transfer of monocarboxylic acid, which can affect the metabolism and activity of cells (10). The expression levels of PFKFB3 and MCT1 were downregulated by their targeting siRNAs, while quercetin treatment only inhibited MCT1 expression but did not affect PFKFB3 expression (Fig. 3A and B).

Knockdown of MCT1 and PFKFB3 increases the apoptosis rate of HUVECs under single-culture and co-culture situations. Under the 3D co-culture condition, the apoptosis rate was slightly lower than that under the single-cultured condition, while the overall trend was similar and without statistical differences (Fig. 4). The apoptosis rate in cells treated with quercetin was significantly increased when compared with that in the control group $(\mathrm{P}<0.05)$. Meanwhile, no significant differences in the apoptosis rate were found among the quercetin, $\mathrm{MCT}^{\mathrm{KD}}$ and $\mathrm{PFKFB} 3^{\mathrm{KD}}$ groups $(\mathrm{P}>0.05)$. In addition, the apoptosis rate was higher in the $\mathrm{M}^{\mathrm{KD}}+\mathrm{P}^{\mathrm{KD}}$ group than that in the single targeting MCT1 or PFKFB3 group $(\mathrm{P}<0.05)$.

Knockdown of MCT1 and PFKFB3 increases the inhibition ratio and decreases the lactate concentration. We next 


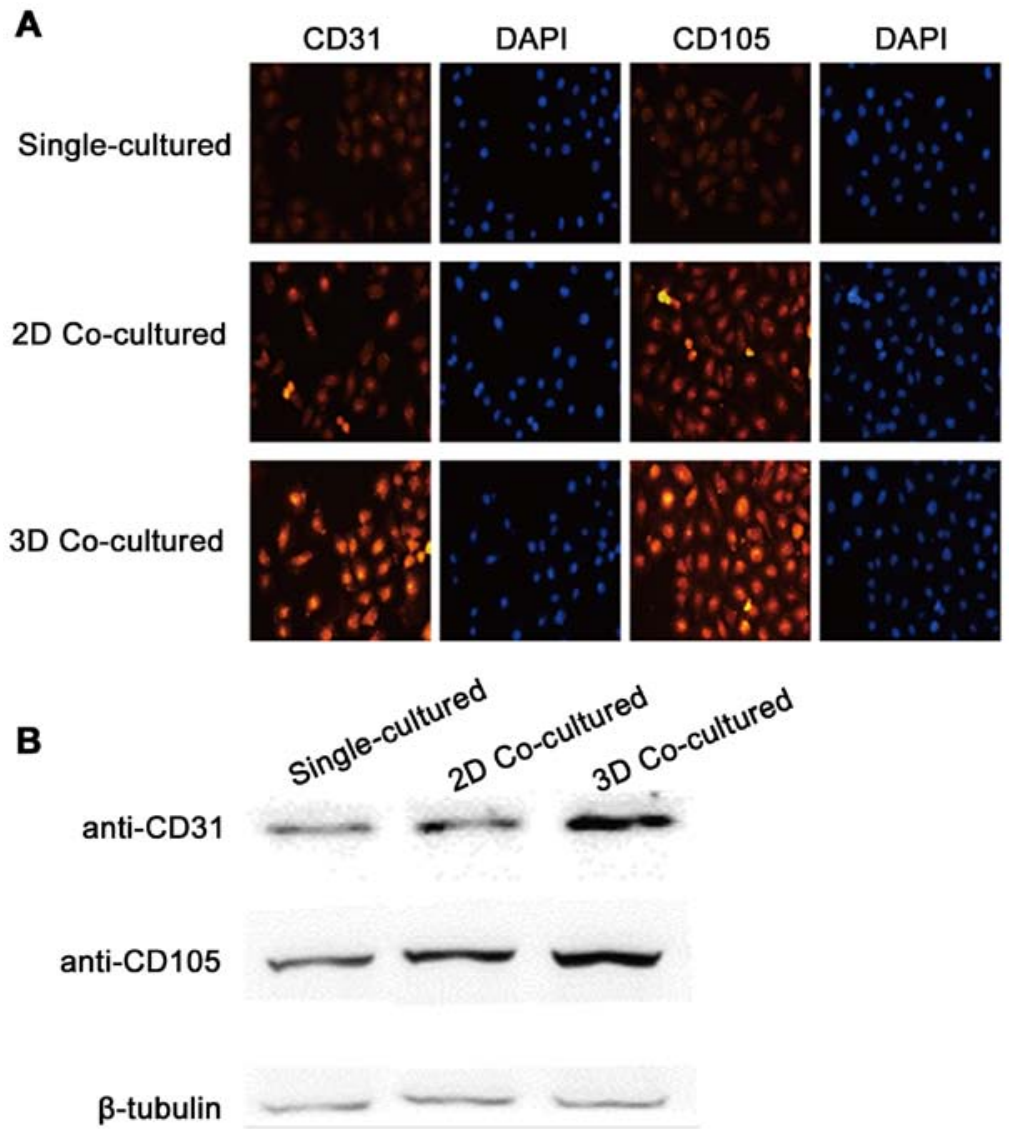

Figure 2. Co-culture of T24 cells and HUVECs enhances HUVEC activation. (A) After co-culture of T24 cells and HUVECs for 3 days, CD31 and CD105 were stained with TRITC and nuclei were stained with DAPI, and examined using a fluorescence microscope (original magnification, x200). (B) CD31 and CD105 expression in the control, 2D and 3D groups as detected by western blot assay.

A

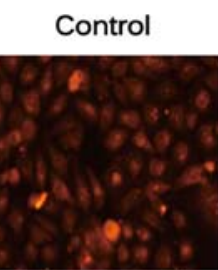

SiPFKFB3

Quercetin

PFKFB3
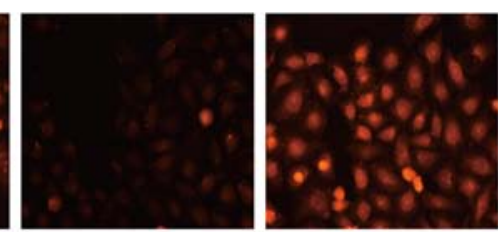

DAPI


B

Control

siMCT1

Quercetin

MCT1
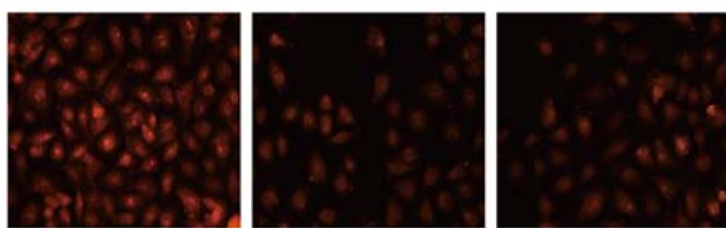

DAPI
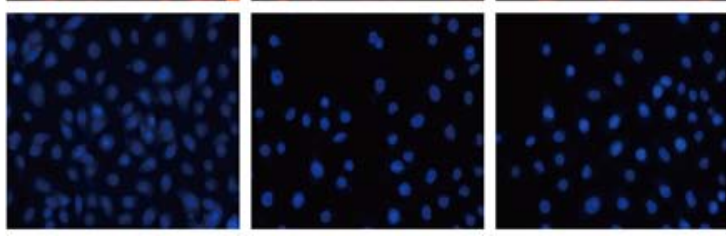

Figure 3. MCT1 and PFKFB3 were inhibited by quercetin and siRNAs. (A) PFKFB3 and (B) MCT1 expression levels were examined using immunofluorescence after cells were treated with siRNAs and quercetin (magnification, x200). 
A
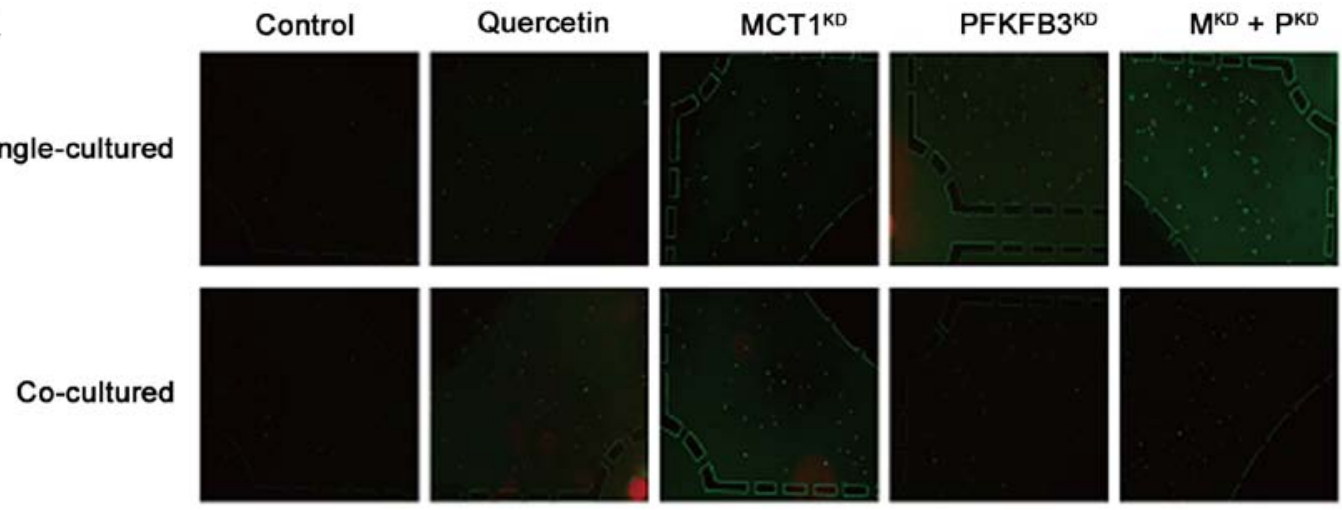

B

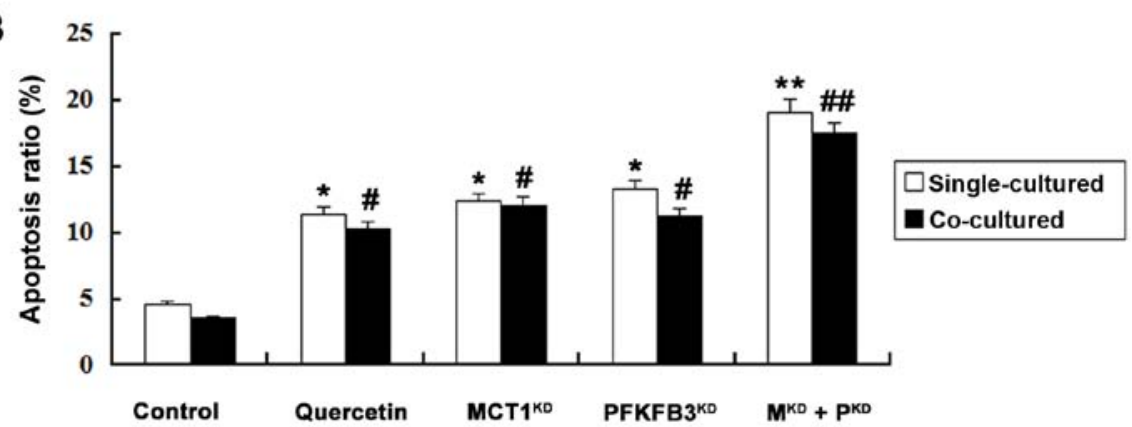

Figure 4. Downregulation of the expression of MCT1 and PFKFB3 increases the apoptosis rate of HUVECs. (A) A chamber was planted with endothelial cells and the cell number was $\sim 300-400 /$ chamber. Cells were divided into 5 groups, namely the control, quercetin (cells were treated with $5 \mu 10.02 \mathrm{nmol} / \mu 1$ quercetin), $\mathrm{MCT}^{\mathrm{KD}}$ (MCT1 was knocked down with $5 \mu 10.02 \mathrm{nmol} / \mu 1$ siMCT1), PFKFB3 ${ }^{\mathrm{KD}}$ (PFKFB3 was knocked down with $5 \mu 10.02 \mathrm{nmol} / \mu 1 \mathrm{siPFKFB} 3$ ) and $\mathrm{M}^{\mathrm{KD}}+\mathrm{P}^{\mathrm{KD}}$ group (MCT1 and PFKFB3 were both knocked down with $2.5 \mu 10.02 \mathrm{nmol} / \mu 1 \mathrm{siMCT} 1$ and $2.5 \mu 10.02 \mathrm{nmol} / \mu 1 \mathrm{siPFKFB}$ ). Control group was treated with the same amount of DMEM. The cells in the different treatment groups were stained with $2 \mu \mathrm{mol} / 1$ calcein and $10 \mu$ mol/l propidium iodide, and then were observed under a microscope (magnification, x200). (B) Statistical results of the apoptosis rate and mortality ratio of HUVECs in the different treatment groups. One-way ANOVA followed by Tukey's post hoc test was used to analyze the differences among groups. Columns, mean $(\mathrm{n}=3)$; bars, $\mathrm{SD}$; ${ }^{*} \mathrm{P}<0.05,{ }^{* *} \mathrm{P}<0.01$ vs. the control group in the single-culture cells; ${ }^{\#} \mathrm{P}<0.05,{ }^{\# \#} \mathrm{P}<0.01$ vs. the control group in the co-culture cells.

A

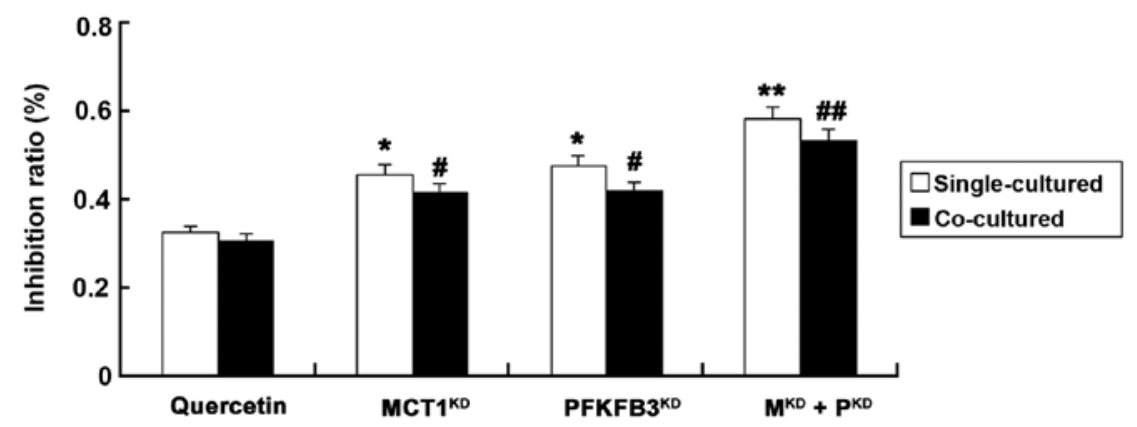

B

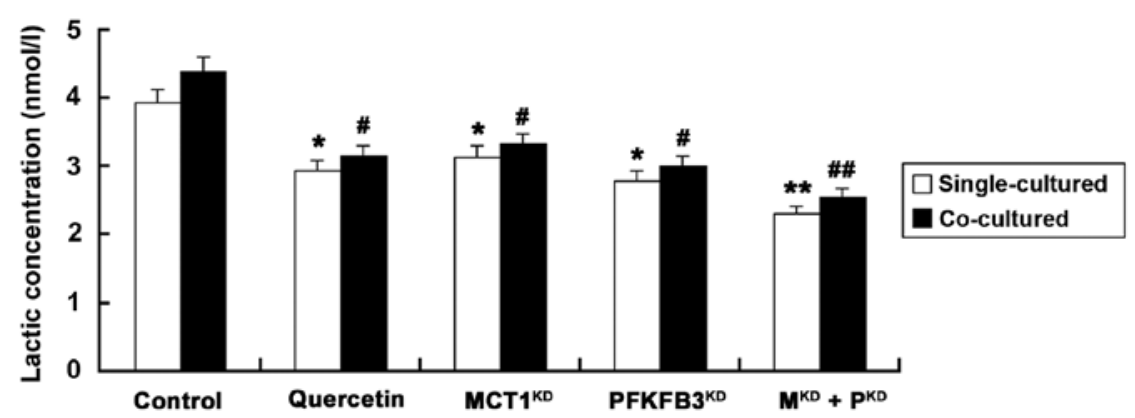

Figure 5. Knockdown of MCT1 and PFKFB3 increases the inhibition ratio and decreases the lactate concentration. Cells were divided into 5 groups, namely the control, quercetin (cells were treated with $5 \mu 10.02 \mathrm{nmol} / \mu 1$ quercetin), MCT1 ${ }^{\mathrm{KD}}$ (MCT1 was knocked down with $5 \mu 10.02 \mathrm{nmol} / \mu 1 \mathrm{siMCT} 1$ ), PFKFB3 ${ }^{\mathrm{KD}}$ (PFKFB3 was knocked down with $5 \mu 10.02 \mathrm{nmol} / \mu 1$ siPFKFB3) and $\mathrm{M}^{\mathrm{KD}}+\mathrm{P}^{\mathrm{KD}}$ groups (MCT1 and PFKFB3 were both knocked down with $2.5 \mu 10.02 \mathrm{nmol} /$ $\mu 1 \mathrm{siMCT} 1$ and $2.5 \mu 10.02 \mathrm{nmol} / \mu 1 \mathrm{siPFKFB} 3$ ). Control group was treated with the same amount of DMEM. All the cells were cultured in microfluidic chips for 3 days to evaluate the suppression ratio (A) and lactic concentration (B) in the different groups. One-way ANOVA followed by Tukey's post hoc test was used to analyze the differences among groups. Columns, mean ( $\mathrm{n}=3$ ); bars, $\mathrm{SD} ;{ }^{*} \mathrm{P}<0.05,{ }^{* *} \mathrm{P}<0.01$ vs. the quercetin (A) or control groups (B) in single-culture cells; ${ }^{~} \mathrm{P}<0.05,{ }^{\# \#} \mathrm{P}<0.01$ vs. the quercetin (A) or control groups (B) in co-cultured cells. 
evaluated the cell proliferation and lactate concentration in the co-cultured HUVECs and T24 cells following the different treatments (Fig. 5). The inhibition ratios were similar whether silencing of MCT1 or PFKFB3 was carried out by siRNAs $(\mathrm{P}>0.05)$, and the ratios were all higher than that in cells treated with the same amount of quercetin $(\mathrm{P}<0.05)$. Additionally, the suppression rate was highest in the $\mathrm{M}^{\mathrm{KD}}+\mathrm{P}^{\mathrm{KD}}$ group. Furthermore, the lactic concentration was significantly reduced in cells following silencing of MCT1 or/and PFKFB3, as compared with that in the control group $(\mathrm{P}<0.05)$.

\section{Discussion}

Endothelial cells (ECs) are usually considered as ideal targets for suppression of tumor angiogenesis owing to various factors. i) Many important receptors on EC membranes take part in angiogenesis; ii) EC structure is stable and it is difficult to form drug resistance; iii) ECs have similar characteristics in almost all solid tumor, thus, one target can be used in different tumor treatments; iv) direct contact between ECs and drugs in blood can reduce drug concentrations (17). ECs tend to form tip cell phenotype to bud blood vessels under a high lactic acid concentration. At the same time, another part of ECs translate into a stalk cell phenotype, obtaining strong proliferation ability leading to new sprout growth into vessel branches (18). All the experiments in the present study were conducted in a simulated tumor microenvironment by co-culturing HUVECs and T24 cells with a microfluidic device.

Recently, several studies have found that CD31 and CD105 are specific sensitive microvessel (MV) markers in various types of cancer, such as colon, cervix, endometrium and breast $(16,19-21)$. We analyzed the fluorescence intensity of CD31 and CD105 in single-, 2D- and 3D-cultured HUVECs, and strong positive expression of CD31 and CD105 was found in the 3D co-cultured cells (Fig. 2). This occurred since the cells were embedded into Matrigel in a 3D condition which was most similar to a real in vivo situation and thus the variety of cellular interactions were most frequent. This result also illustrated that tumor cells could enhance EC activity.

MCT1 is an important transporter for lactic acid entering ECs and plays an important role in the presence of lactic acid (22). PFKFB3, acting as a PFK1 allosteric activator, plays an important role in glycolysis. Carmeliet et al confirmed that glycolysis is the important source for ECs to gain energy and build new blood vessels (23). Meanwhile, research indicates that blood vessel growth is inhibited by blocking glycolysis and removing the energy source of vascular ECs. Targeting PFKFB3 can effectively reduce the motility, proliferation, invasion and angiogenesis of ECs (24). In the present study, the apoptosis rate of the HUVECs was significantly increased after silencing of MCT1 or/and PFKFB3 (Fig. 4). The possible reason may be that silencing of MCT1 or/and PFKFB blocked the main energy metabolic pathway, which accounts for $\sim 85 \%$ of the energy of ECs (25).

Rivera and Bergers found that PFKFB3 promoted EC proliferation by reducing Notch pathway activity (26). Végran et al reported that lactate could enter ECs through MCT-1 to trigger the phosphorylation and degradation of I $\kappa \mathrm{B} \alpha$, and then stimulate the NF- $\kappa \mathrm{B}$ pathway to drive cell migration and tube formation in colorectal adenocarcinoma and breast cancer (27). CCK-8 assay verified that blocking MCT1 or PFKFB3 reduced the proliferation activity of HUVECs and the combined blockage of the targets provided a better result (Fig. 5). Single blockage of PFKFB3 was found to only partly and transitorily reduce EC activity, since the lactic acid from other cells in the microenvironment can resist this effect (28). Multipoint target blocking is considered as the most effective method for inhibiting tumor cell proliferation (29). The effects on apoptosis and proliferation were much stronger following the combination of blocking MCT1 and PFKFB3 when compared with the effects following either blocking MCT1 or PFKFB3. Thus, we speculated that the energy metabolic pathway and lactic acid effects may be involved.

As the end-product of metabolism, lactate acid remains stable and high concentrations in the tumor microenvironment stimulate angiogenesis (30). It has been reported that high serum lactate dehydrogenase levels are considered as a poor prognostic indicator in most types of cancers, including pancreatic carcinoma, malignant lymphoma and colorectal cancers (31-33). It has been reported that lactic acid exists as the substrate of many types of enzymes in tumor ECs, and an increase in its concentration could promote angiogenesis by promoting the activities of NF- $\mathrm{KB}$ and IL-8/CXCL8 signaling pathways by inhibiting PDH2 (6). In the present study, the lactate concentration was significantly decreased by silencing MCT1 or/and PFKFB3. However, the underlying mechanisms involved in the effects of MCT1 and PFKFB3 on lactate concentration in $\mathrm{BC}$ warrant further study.

Research has demonstrated that angiogenesis, proliferation and metastatic activity of cancers could be effectively inhibited by targeting MCT1 and PFKFB3 (34-36). Quercetin can be used as an anti-cough drug and is a good expectorant. Recently, quercetin was found to have an inhibitory effect on MCT1 activity, thus promoting cell apoptosis (37). According to our results, quercetin effectively suppressed cell proliferation and promoted apoptosis via a significant decrease in the activity of MCT1 and downregulation of lactic acid concentration may be involved in these effects in BC. Considering that no obvious differences in effects were found between quercetin and siMCT1, we can conclude that quercetin has potential antitumor effects by targeting MCT1.

In conclusion, the present study demonstrated that tumor cells enhance EC activity under a simulated microenvironment by 3D co-culture of HUVECs and T24 cells. Meanwhile, cell apoptosis increased and proliferation decreased following the blocking of MCT1 or/and PFKFB3 via influencing the energy metabolic pathway and lactic acid concentration, which are critical for angiogenesis. Quercetin may be developed as a potential antitumor drug by downregulating MCT1. However, further studies should be carried out to investigate the molecular mechanisms of quercetin in regards to the effect on MCT1.

\section{Acknowledgements}

The present study was supported by a grant from the National Natural Science Foundation of China (nos. 30901481, 81372752 and 81472411), the Wu Jieping Medical Foundation (320.6750.13261), and the Natural Science Foundation of Shandong Province, China (ZR2014HM088). 
We thank Professor Chang Gui Li (Qingdao University, China) for providing the Gout Laboratory. We also thank Professor Zhen Liu and Ling Ling Cui for providing technical guidance.

\section{References}

1. Jemal A, Bray F, Center MM, Ferlay J, Ward E and Forman D: Global cancer statistics. CA Cancer J Clin 61: 69-90, 2011.

2. Sleeckx N, Van Brantegem L, Van den Eynden G, Fransen E, Casteleyn C, Van Cruchten S, Veldhuis Kroeze E and Van Ginneken C: Angiogenesis in canine mammary tumours: A morphometric and prognostic study. J Comp Pathol 150: 175-183, 2014.

3. Müller K, Ellenberger C, Hoppen HO and Schoon HA: Immunohistochemical study of angiogenesis and angiogenic factors in equine granulosa cell tumours. Res Vet Sci 92: 471-477, 2012.

4. Hoelder S, Clarke PA and Workman P: Discovery of small molecule cancer drugs: Successes, challenges and opportunities. Mol Oncol 6: 155-176, 2012.

5. Ngo H, Tortorella SM, Ververis K and Karagiannis TC: The Warburg effect: Molecular aspects and therapeutic possibilities. Mol Biol Rep 42: 825-834, 2015

6. Bonavia R, Inda MM, Vandenberg S, Cheng SY, Nagane M, Hadwiger P, Tan P, Sah DW, Cavenee WK and Furnari FB: EGFRvIII promotes glioma angiogenesis and growth through the NF- $\kappa B$, interleukin-8 pathway. Oncogene 31: 4054-4066, 2012.

7. Philip B, Ito K, Moreno-Sánchez R and Ralph SJ: HIF expression and the role of hypoxic microenvironments within primary tumours as protective sites driving cancer stem cell renewal and metastatic progression. Carcinogenesis 34: 1699-1707, 2013.

8. De Bock K, Georgiadou M, Schoors S, Kuchnio A, Wong BW, Cantelmo AR, Quaegebeur A, Ghesquière B, Cauwenberghs $S$ Eelen G, et al: Role of PFKFB3-driven glycolysis in vessel sprouting. Cell 154: 651-663, 2013.

9. Doherty JR and Cleveland JL: Targeting lactate metabolism for cancer therapeutics. J Clin Invest 123: 3685-3692, 2013.

10. Izumi H, Takahashi M, Uramoto H, Nakayama Y, Oyama T, Wang KY, Sasaguri Y, Nishizawa S and Kohno K: Monocarboxylate transporters 1 and 4 are involved in the invasion activity of human lung cancer cells. Cancer Sci 102: 1007-1013, 2011.

11. Ge X, Lyu P, Cao Z, Li J, Guo G, Xia W and Gu Y: Overexpression of miR-206 suppresses glycolysis, proliferation and migration in breast cancer cells via PFKFB3 targeting. Biochem Biophys Res Commun 463: 1115-1121, 2015.

12. Bokhari M, Carnachan RJ, Cameron NR and Przyborski SA: Culture of HepG2 liver cells on three dimensional polystyrene scaffolds enhances cell structure and function during toxicological challenge. J Anat 211: 567-576, 2007.

13. Knight E and Przyborski S: Advances in 3D cell culture technologies enabling tissue-like structures to be created in vitro. J Anat 227: 746-756, 2015.

14. Huh D, Matthews BD, Mammoto A, Montoya-Zavala M, Hsin HY and Ingber DE: Reconstituting organ-level lung functions on a chip. Science 328: 1662-1668, 2010

15. Shi H, Jiang H, Wang L, Cao Y, Liu P, Xu X, Wang Y, Sun L and Niu H: Overexpression of monocarboxylate anion transporter 1 and 4 in T24-induced cancer-associated fibroblasts regulates the progression of bladder cancer cells in a 3D microfluidic device. Cell Cycle 14: 3058-3065, 2015.

16. Saad RS, El-Gohary Y, Memari E, Liu YL and Silverman JF: Endoglin (CD105) and vascular endothelial growth factor as prognostic markers in esophageal adenocarcinoma. Hum Pathol 36: 955-961, 2005.

17. Kim C, Yang H, Fukushima Y, Saw PE, Lee J, Park JS, Park I, Jung J, Kataoka H, Lee D, et al: Vascular RhoJ is an effective and selective target for tumor angiogenesis and vascular disruption. Cancer Cell 25: 102-117, 2014

18. Eelen G, Cruys B, Welti J, De Bock K and Carmeliet P: Control of vessel sprouting by genetic and metabolic determinants. Trends Endocrinol Metab 24: 589-596, 2013.
19. Akagi K, Ikeda Y, Sumiyoshi Y, Kimura Y, Kinoshita J, Miyazaki $M$ and Abe T: Estimation of angiogenesis with antiCD105 immunostaining in the process of colorectal cancer development. Surgery 131 (Suppl): S109-S113, 2002.

20. Saad R and Dabbs D: Endoglin, CD31, and CD34 expression in the breast cancer. Laboratory Investigation. Nature Publishing Group, New York, NY, USA, 2001.

21. Saad RS, Jasnosz KM, Tung MY and Silverman JF: Endoglin (CD105) expression in endometrial carcinoma. Int J Gynecol Pathol 22: 248-253, 2003.

22. Su J, Chen X and Kanekura T: A CD147-targeting siRNA inhibits the proliferation, invasiveness, and VEGF production of human malignant melanoma cells by down-regulating glycolysis. Cancer Lett 273: 140-147, 2009.

23. Carmeliet P, De Smet F, Loges S and Mazzone M: Branching morphogenesis and antiangiogenesis candidates: Tip cells lead the way. Nat Rev Clin Oncol 6: 315-326, 2009.

24. Xu Y, An X, Guo X, Habtetsion TG, Wang Y, Xu X, Kandala S, Li Q, Li H, Zhang C, et al: Endothelial PFKFB3 plays a critical role in angiogenesis. Arterioscler Thromb Vasc Biol 34: 1231-1239, 2014

25. Stapor P, Wang X, Goveia J, Moens S and Carmeliet P: Angiogenesis revisited - role and therapeutic potential of targeting endothelial metabolism. J Cell Sci 127: 4331-4341, 2014.

26. Rivera LB and Bergers G: Angiogenesis. Targeting vascular sprouts. Science 344: 1449-1450, 2014.

27. Végran F, Boidot R, Michiels C, Sonveaux P and Feron O: Lactate influx through the endothelial cell monocarboxylate transporter MCT1 supports an NF- $\mathrm{KB} / \mathrm{IL}-8$ pathway that drives tumor angiogenesis. Cancer Res 71: 2550-2560, 2011.

28. Schoors S, Cantelmo AR, Georgiadou M, Stapor P, Wang X, Quaegebeur A, Cauwenberghs S, Wong BW, Bifari F, Decimo I, et al: Incomplete and transitory decrease of glycolysis: A new paradigm for anti-angiogenic therapy? Cell Cycle 13: 16-22, 2014.

29. Niu H, Jiang H, Cheng B, Li X, Dong Q, Shao L, Liu S and Wang X: Stromal proteome expression profile and muscleinvasive bladder cancer research. Cancer Cell Int 12: 39, 2012.

30. Tennant DA, Durán RV and Gottlieb E: Targeting metabolic transformation for cancer therapy. Nat Rev Cancer 10: 267-277, 2010.

31. Yi JH, Kim JH, Baek KK, Lim T, Lee DJ, Ahn YC, Kim K, Kim SJ, Ko YH and Kim WS: Elevated LDH and paranasal sinus involvement are risk factors for central nervous system involvement in patients with peripheral T-cell lymphoma. Ann Oncol 22: 1636-1643, 2011.

32. Fahmueller YN, Nagel D, Hoffmann RT, Tatsch K, Jakobs T, Stieber P and Holdenrieder S: Predictive and prognostic value of circulating nucleosomes and serum biomarkers in patients with metastasized colorectal cancer undergoing Selective Internal Radiation Therapy. BMC Cancer 12: 5, 2012.

33. Tas F, Aykan F, Alici S, Kaytan E, Aydiner A and Topuz E: Prognostic factors in pancreatic carcinoma: Serum LDH levels predict survival in metastatic disease. Am J Clin Oncol 24: 547-550, 2001.

34. Murray CM, Hutchinson R, Bantick JR, Belfield GP, Benjamin AD, Brazma D, Bundick RV, Cook ID, Craggs RI, Edwards S, et al: Monocarboxylate transporter MCT1 is a target for immunosuppression. Nat Chem Biol 1: 371-376, 2005.

35. Vander Heiden MG: Targeting cancer metabolism: A therapeutic window opens. Nat Rev Drug Discov 10: 671-684, 2011.

36. Le Floch R, Chiche J, Marchiq I, Naiken T, Ilc K, Murray CM, Critchlow SE, Roux D, Simon M-P and Pouysségur J: CD147 subunit of lactate $/ \mathrm{H}^{+}$symporters MCT1 and hypoxia-inducible MCT4 is critical for energetics and growth of glycolytic tumors. Proc Natl Acad Sci USA 108: 16663-16668, 2011.

37. Chao JI, Su WC and Liu HF: Baicalein induces cancer cell death and proliferation retardation by the inhibition of CDC2 kinase and survivin associated with opposite role of p38 mitogen-activated protein kinase and AKT. Mol Cancer Ther 6: 3039-3048, 2007. 